\title{
CMTM8 inhibits the carcinogenesis and progression of bladder cancer
}

\author{
DENGHUI GAO ${ }^{1 *}$, HAO HU $^{1 *}$, YING WANG $^{2}$, WEIDONG YU ${ }^{1}$, JIANHUA ZHOU $^{1}$, \\ XIAOFENG WANG ${ }^{1}$, WEIPING WANG ${ }^{3}$, CHUNYAN ZHOU $^{3}$ and KEXIN XU ${ }^{1}$
}

\author{
${ }^{1}$ Department of Urology, Peking University People's Hospital; ${ }^{2}$ Department of Immunology, School of Basic \\ Medical Sciences; Key Laboratory of Medical Immunology of the Ministry of Health, Peking University; \\ ${ }^{3}$ Department of Biochemistry and Molecular Biology, School of Basic Medical Sciences; Key Laboratory of \\ Molecular Cardiovascular Sciences, the Ministry of Education of China, Peking University, Beijing, P.R. China
}

Received June 25, 2015; Accepted August 7, 2015

DOI: $10.3892 /$ or.2015.4310

\begin{abstract}
Bladder cancer is the most common tumor of the urinary tract. The incidence of bladder cancer has increased in the last few decades, thus novel molecular markers for early diagnosis and more efficacious treatment are urgently needed. Chemokine-like factor (CKLF)-like MARVEL transmembrane domain containing 8 (CMTM8) is downregulated in several types of cancers and is associated with tumor progression. However, CMTM8 expression has been unexplored in bladder cancer to date. Our results revealed that the expression of CMTM8 was negative in 46 of 74 (62.2\%) bladder cancer samples via immunohistochemistry assay. CMTM8 downregulation was associated with advancing tumor stage and tumor grade. CMTM8 was successfully overexpressed by lentivirus in EJ and T24 cells, and the CCK-8 and Transwell assays showed that CMTM8 overexpression decreased cell proliferation, migration and invasion in vitro. In tumor xenografts upregulation of CMTM8 inhibited tumor growth and lymph node metastasis in vivo. In conclusion, overexpression of CMTM8 in bladder cancer results in reduced malignant cell growth, migration and invasion, which could make it a potential therapeutic target in the treatment of bladder cancer.
\end{abstract}

\section{Introduction}

Bladder cancer is the most common tumor of the urinary tract, with an estimated 429,800 new cases and 165,100 deaths occurring worldwide each year (1). There is considerable global

Correspondence to: Dr Kexin Xu, Department of Urology, Peking University People's Hospital, 11 Xizhimen Street, Xicheng, Beijing, P.R. China

E-mail: cavinx@sina.com

${ }^{*}$ Contributed equally

Key words: bladder cancer, CMTM8, proliferation, invasion, migration variation in the incidence due to differences in risk factors such as tobacco use, Schistosoma infection, chemical exposure, diet and lifestyle trends, atmospheric pollution and genetic susceptibilities $(2,3)$. In Western countries, urothelial carcinoma accounts for $90 \%$ of the total incidence, while squamous cell carcinoma is prevalent in Africa and the Middle East (4). In China, it has been reported that bladder cancer is the most common genitourinary malignancy, and the incidence of this disease has increased in the last few decades (5). Despite the sophistication of surgical techniques and adjuvant therapies, 5 -year survival rates are only $\sim 60 \%$ (6). Furthermore, these tumors have a $30-70 \%$ chance of recurrence, and quickly progress to muscle-invasive disease in up to $30 \%$ of the population (7). Therefore, novel molecular markers for the early diagnosis and more efficacious treatment are urgently needed for the benefit of bladder cancer patients.

The chemokine-like factor superfamily (CKLFSF) represents a protein family of cytokines that is different from classical cytokines because of dissimilar amino acid sequences (8). CKLF-like MAL-related proteins for vesicle trafficking and membrane link transmembrane domain (MARVEL) containing 8 (CMTM8) formerly known as chemokine-like factor superfamily 8 (CKLFSF8) was isolated and cloned from PHA-stimulated histiocytic lymphoma cells $(8,9)$. Out of the two isoforms of CMTM8, the long isoform is the one which is predominantly expressed in human cell lines as well as in normal human tissues $(10,11)$.

Many authors have reported low expression of CMTM8 in esophageal, cardiac (http://en.cnki.com.cn/Article en/CJFDTOTAL-SDYY200906013.htm), low-grade clear-cell renal cell carcinoma (12) and brain metastatic triple-negative breast carcinoma (13). A previous study demonstrated that downregulation of CMTM8 induced epithelial-to-mesenchymal transition-like changes via c-MET/extracellular signal-regulated kinase (ERK) signaling in HepG2 hepatocellular carcinoma cells (14). Overexpression of CMTM8 attenuated or even inhibited EGFR downstream signaling by ligand-receptor mediated internalization and associated desensitization $(15,16)$. Further studies also found that CMTM8, a negative regulator of EGF-induced signaling, decreased levels of Bad-phosphorylation and promoted apoptosis through 
caspase-dependent and -independent pathways (17). EGFR is an important growth factor receptor thoroughly studied in bladder cancer. It is activated in the presence of other growth factors or ligands, leading to homo- or hetero-dimerization with another EGFR, and activation of signaling pathways such as MAPK and Akt involved in cell survival and proliferation (18). The expression of EGFR is highly expressed in the cancerous bladder compared to normal bladder (19-21). Hence, although debates exist, EGFR expression correlates with a higher risk and mortality in bladder cancer. These studies suggest a link with CMTM8, which may exhibit its function by suppressing tumor growth via inhibiting EGFR during cancer progression.

We thus proposed the utility of CMTM8 as a prognostic biomarker in the prediction of bladder cancer progression, as well as its manipulation in the treatment of bladder cancer. To date, the expression profile of CMTM8 in human bladder cancer and its biological role remain unclear. In the present study, we examined the expression pattern of CMTM8 protein in 74 patients with bladder cancer using immunohistochemistry, and analyzed its correlation with clinicopathological factors. Furthermore, we overexpressed CMTM8 in bladder cancer cells, and explored the biological effects of CMTM8 that may impact bladder tumor growth.

\section{Materials and methods}

Patients and samples. The protocol utilized in this study was approved by the Peking University Institutional Review Board. Primary tumor specimens were obtained from 74 patients diagnosed with bladder cancer who underwent resection at the People's Hospital of Peking University between 2008 and 2010. The histological diagnosis and tumor grades were evaluated for sections stained with $H \& E$ according to the WHO classification guidelines. Tumors were classified into Ta, T1, T2, T3 and T4 according to WHO guidelines (2007). The study was regulated by the Ethics Reviewing Committee of Peking University People's Hospital, China.

Immunohistochemical analysis. Immunohistochemistry was performed on human bladder cancer tissues to detect the expression of CMTM8. Briefly, formalin-fixed and paraffin wax-embedded tissues were cut into thin histologic sections, and fixed onto slides. The sections were deparaffinized with xylene and decreasing grades of alcohol. This was followed by antigen retrieval $(15 \mathrm{~min})$ with trypsin, and addition of $3 \% \mathrm{H}_{2} \mathrm{O}_{2}$ to quench endogenous peroxidase activity. After washing and rinsing steps with PBS, the slides were blocked with $10 \%$ normal goat serum (Zhongshan Jinqiao Co., Beijing, China), and incubated with the polyclonal rabbit CMTM8 antibody (Peking University Human Disease Genomics Research Center, China) overnight at $4^{\circ} \mathrm{C}$. On the following day, the slides were incubated with the secondary antibody (30 min). The slides were washed again, and the antigen-antibody complex was detected by DAB (Xiyajinqiao, Beijing, China).

The staining intensity was categorized as follows: 0 , negative; 1 , weak; and 2, strong. The percentage of stained tumor cells was scored as $0,0 \% ; 1,1-25 \% ; 2,26-50 \% ; 3,51-75 \%$; and $4,76-100 \%$. The scores for each tumor sample were multiplied to give a final score of $0-8$; the tumor samples with a final score $<4$ were regarded as negative, and tumor samples with a final score of 4-8 were determined to be CMTM8 positive (22).

Cell culture. RT4, 5637 and T24 cell lines were obtained from ATCC (Manassas, VA, USA). EJ cell lines were obtained as a gift from Peking University Institute of Urology. The cells were cultured in RPMI-1640 (EJ, 5637 and T24) and McCoy's 5A (RT4) media (Gibco Life Technologies, Carlsbad, CA, USA) containing $10 \%$ fetal bovine serum (FBS) (Invitrogen Life Technologies, Carlsbad, CA, USA), 100 IU/ml penicillin (Solarbio, Beijing, China) and $100 \mu \mathrm{g} / \mathrm{ml}$ streptomycin (Solarbio) under a $5 \% \mathrm{CO}_{2}$ atmosphere.

Western blot analysis. Protein was extracted using lysis buffer (50 mM Tris-HCl, pH 7.4, $150 \mathrm{mM} \mathrm{NaCl,} \mathrm{1 \%} \mathrm{NP-40}$ and $0.1 \%$ sodium dodecyl sulfate), and the concentration was measured using the BCA protein assay kit (Pierce, Rockford, IL, USA) using bovine serum albumin as the standard. Proteins were run on SDS-PAGE gels $(12 \%)$ and electro-transferred onto a nitrocellulose membrane at $4^{\circ} \mathrm{C}$ for $30 \mathrm{~min}$. Thereafter, the blots were probed with anti-CMTM8 (1:500; Proteintech, Wuhan, China) and anti-GAPDH (1:2000; Emarbio, Beijing, China) overnight at $4^{\circ} \mathrm{C}$, followed by incubation with horseradish peroxidase-conjugated secondary antibody (Santa Cruz Biotechnology, Inc., Santa Cruz, CA, USA) at room temperature for $2 \mathrm{~h}$. Proteins were detected by a chemiluminescence kit according to the manufacturer's recommendations (ECL; Millipore, Billerica, MA, USA). GAPDH was used as the internal control.

$R T-P C R$. Total RNA was isolated from cells using TRIzol reagent (Invitrogen Life Technologies), according to the manufacturer's instructions. cDNA synthesis was carried out in a $25 \mu \mathrm{l}$ reaction mixture containing $2 \mu \mathrm{g}$ total RNA, $400 \mathrm{mM}$ reverse transcription primers, $4 \mathrm{U} / \mu 1 \mathrm{M}-\mathrm{MLV}$, $1 \mathrm{U} / \mu \mathrm{l}$ RNasin and $0.4 \mathrm{mM}$ dNTP mix, using M-MLV reverse transcriptase (Promega, Madison, WI, USA). The amplification reaction was carried out in an ABI-7300 real-time PCR system (Applied Biosystems, Foster City, CA, USA) with SYBR-Green Master Mix (Toyobo, Osaka, Japan). The annealing temperature was $60^{\circ} \mathrm{C}$. Transcription levels were normalized against $18 \mathrm{~S}$, and the data are representative of the average of three independent experiments. The sequences of primers for amplification were as follows: CMTM8 forward, 5-GGAGGAGCCGCAGCGCG-3 and CMTM8 reverse, 5-CTGTATGGTCCTGGATCTCC-3; 18S forward, 5-ACCACAGTCCATGCCATCAC-3 and 18S reverse, 5-TCC ACCACCCTGTTGCTGTA-3.

Recombinant lentivirus construction and transfection. The human CMTM8 gene fragments were amplified by polymerase chain reaction. The primers for PCR were synthesized as follows: F, AAAGAATTCATGGAGGAGCCGCAGCGC and R, AAAGGATCCTCACTGT. Recombinant lentiviruses, which coexpress enhanced green fluorescent protein (GFP) and the CMTM8 sequence, were produced in 293T cells. Lentiviral-expressing green GFP was generated as a control (Genechem, Shanghai, China).

T24 and EJ cells in log phase growth were cultured in 6 -well plates at a density of $5 \times 10^{4}$ cells/well and transfected 
A

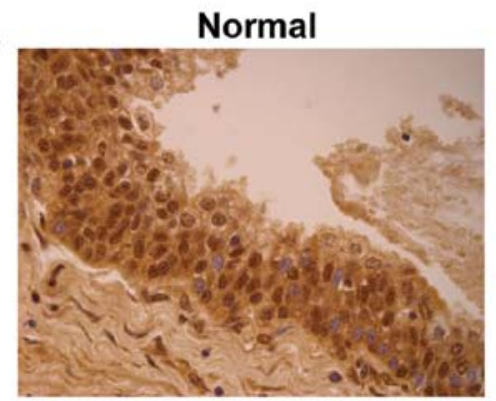

B

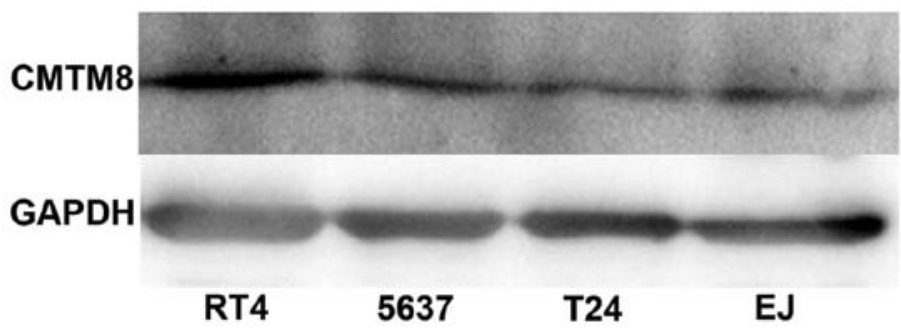

Low grade

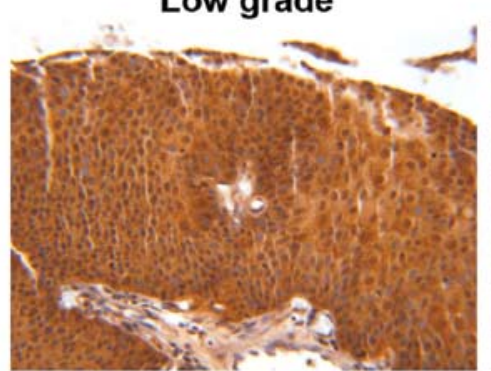

High grade

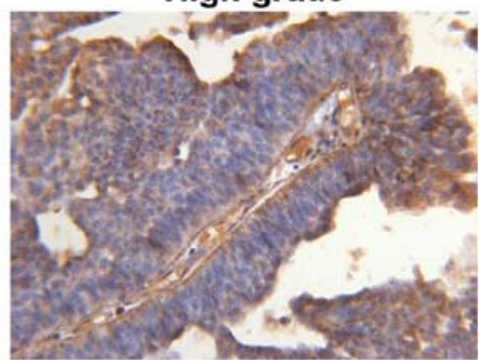

C

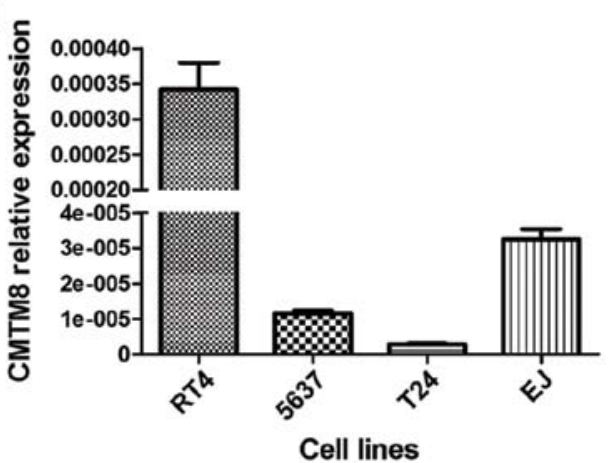

Figure 1. CMTM8 is downregulated in human bladder carcinoma tissues and bladder cancer cell lines. (A) The expression of CMTM8 in normal and cancerous (low-grade/high-grade) human bladder tissues as obtained by immunohistochemistry (magnification, x400). As indicated, normal and low-grade cancerous bladders showed high expression of CMTM8, while negative or weaker staining of CMTM8 was observed in the high-grade bladder cancers. (B) CMTM8 expression was detected by western blotting in low-grade (RT4) as well as high-grade (5637, T24 and EJ) bladder cancer cells. Expression of CMTM8 was found to be high in the low-grade tumor cell lines, while it was significantly less in the high-grade bladder cancer cell lines. GAPDH was used as the loading control. (C) Quantitative real-time PCR experiments showing mRNA expression of CMTM8 in low- and high-grade bladder cancer cell lines. Elevated levels of CMTM8 mRNA was noted in the RT4 cells, while all other cell lines had low expression.

with the CMTM8-GFP vectors (CMTM8 overexpression group) or the GFP vectors (expressing GFP) at a multiplicity of infection (MOI) of 20 in serum-free media. After incubation at $37^{\circ} \mathrm{C}$ for $16 \mathrm{~h}$, the transduction medium was replaced with fresh RPMI-1640 + 10\% FBS. CMTM8-T24/EJ cells genetically engineered with a recombinant lentivirus coexpressing GFP and CMTM8 were used as the CMTM8 group, and GFP-T24/EJ cells manipulated with a lentivirus expressing GFP were used as the control group. T24/EJ cells without any manipulation served as the wild-type group, and were cultured as mentioned above. After three days of transfection, GFP was observed using fluorescence microscopy (MicroPublisher 3.3 RTV; Olympus, Tokyo, Japan). The efficiency of transduction was determined by analyzing GFP expression using fluorescence microscopy (IVIS Spectrum; Caliper Life Science, Hopkinton, MA, USA), western blotting and RT-PCR. We also screened T24 cell lines stably expressing CMTM8.

CCK-8 assay for cell viability. Cancer cells were plated in 96-well plates in media containing $10 \% \mathrm{FBS}$ at $\sim 1-3 \times 10^{3}$ cells per well $48 \mathrm{~h}$ after transfection. For quantitation of cell viability, cells were stained using Cell Counting Kit-8 (CCK-8, Dojindo. Molecular Technologies, Inc., Kumamoto, Japan) assays 4 days later. In brief, $10 \mu \mathrm{l}$ of CCK-8 solution was added to each well and incubated for $2 \mathrm{~h}$ at $37^{\circ} \mathrm{C}$. Thereafter, each solution was measured using a microplate reader (Bio-Rad Laboratories Inc., Tokyo, Japan) at $450 \mathrm{~nm}$.

Colony formation assay. T24/EJ cell lines stably expressing CMTM8 were plated at a cell density of $1 \times 10^{3}$ per well onto 6-well culture dishes, and incubated for 2 weeks. The plates were washed and stained with crystal violet. The number of colonies consisting of $>50$ cells was counted using a microscope.

Migration and invasion assays. The invasion assay with Matrigel-coated membranes, and the migration assay without Matrigel-coated membranes were performed using 24-well Transwell inserts ( $8-\mu \mathrm{m}$ pore filters; Corning, Shanghai, China), according to the manufacturer's instructions. The exponential phase cells were harvested and resuspended $\left(5-8 \times 10^{4}\right.$ cells $\left./ \mathrm{ml}\right)$ in serum-free media, seeded and resuspended in $100 \mu \mathrm{l}$ culture medium in the upper chamber. Next, culture medium with $20 \%$ FBS was placed in the bottom well. The Transwells were incubated at $37^{\circ} \mathrm{C}$ in a $5 \% \mathrm{CO}_{2}$ atmosphere for a period of $24 \mathrm{~h}$. The T24 and EJ cells were allowed to migrate through a porous surface to the bottom of the membrane. After incubation, the cells on the bottom of the membrane were fixed with $4 \%$ paraformaldehyde and stained with crystal violet. The number of invading cells or migrating cells on each membrane was determined by microscopy at a x100 magnification, and the number of cells per field was calculated. All the assays were repeated at least three times.

Tumor xenografts and GFP-tagged bladder cancer in vivo imaging. Fifteen BALB/c nude mice were divided randomly into three groups for tumor xenografts: wild-type, lenti-GFP-NC and lenti-GFP-CMTM8 group. Cells were injected subcutaneously into the flanks of mice at a dose of $5 \times 10^{6} / 100 \mu \mathrm{l}$ for each point, three points each. On day 15 
and 29, mice belonging to the lenti-GFP-control group and lenti-GFP-CMTM8 group were anesthetized by isoflurane, and live imaging was performed using IVIS Spectrum with an excitation filter of $465 \mathrm{~nm}$ and an emission filter of $520 \mathrm{~nm}$. The value for the region of interest (ROI) was calculated and plotted.

Statistical analysis. SPSS version 20.0 for Windows was used for all statistical analyses. A $\chi^{2}$ test was used to examine possible correlations between CMTM8 expression and clinicopathologic factors. All P-values are based on a two-sided statistical analysis, and $\mathrm{P}<0.05$ was considered to indicate a statistically significant difference.

\section{Results}

Downregulation of CMTM8 is associated with increased tumor stage and grade in bladder cancer tissues and cell lines. Bladder cancer tissues from 74 patient samples were analyzed for the expression of CMTM8 by immunohistochemistry. As observed, low expression of CMTM8 was correlated with increasing tumor grade and TNM stage (Table I). A total of $21 / 41(51.22 \%, \mathrm{P}<0.01)$ samples were negative for CMTM8 in tissues of Ta-T1 tumor stage, while negativity was more predominant in T2-T4 stage $(29 / 33 ; 87.88 \%, \mathrm{P}<0.01)$. As for tumor grade, $8 / 23(34.78 \%)$ samples were negative for the expression of CMTM8 in the low-grade category, while 41/51 $(80.39 \%$; $\mathrm{P}<0.01)$ samples showed negligible expression in the high-grade category. As for other characteristics such as age and gender, there was no statistically significant correlation which could be deduced from the study. Immunohistochemical staining from representative cancer/normal bladder samples are shown in Fig. 1A. Normal bladder exhibited high expression of CMTM8 in the cytoplasm as well as the nucleus, while low-grade tumor samples also showed high expression. In comparison, high-grade bladder tumors exhibited more negative CMTM8-staining cells. Next, the protein expression of CMTM8 was analyzed by western immunoblotting in bladder cancer cell lines. The results revealed that CMTM8 expression was well correlated with the aggressive nature of the disease (Fig. 1B). RT4 cells, a low-grade papillary bladder cancer cell line showed high expression of CMTM8, while the high-grade and invasive cancer cell lines displayed weak CMTM8 expression. These results were consistent at the transcription levels as observed by the mRNA expression of CMTM8 in these bladder cancer cell lines (Fig. 1C).

Ectopic overexpression of CMTM8 in bladder cancer cells. T24 and EJ cells were transiently overexpressed with CMTM8-GFP or empty vectors in order to determine the impact of the overexpression of CMTM8 on bladder cancer growth. High intensity bands for CMTM8 were obtained by the western blotting for the CMTM8-overexpressing T24 cells compared to the T24 wild-type or T24-empty vector-transfected cells (Fig. 2A). A similar observation was also observed for mRNA levels in the CMTM8-overexpressing T24 cells (Fig. 2B). Moderate overexpression of CMTM8 was also observed in the EJ cells transfected with the CMTM8-GFP vector, compared to the wild-type and empty vector-transfected cells (Fig. 2C). mRNA levels were also elevated upon overexpression of the
Table I. Relationship between CMTM8 expression and clinicopathological characteristics of the bladder cancer cases.

\begin{tabular}{lcccc}
\hline & \multicolumn{3}{c}{ CMTM8 expression } & \\
\cline { 3 - 4 } & $\begin{array}{c}\text { Total } \\
\text { Characteristics }\end{array}$ & $\begin{array}{c}\text { Positive } \\
(\mathrm{N}=74)\end{array}$ & $\begin{array}{c}\text { Negative } \\
(\mathrm{n}=46)\end{array}$ & P-value \\
\hline Age (years) & & & & 0.723 \\
$>55$ & 58 & 19 & 39 & \\
$\leq 55$ & 16 & 6 & 10 & \\
Gender & & & & 0.814 \\
Male & 55 & 19 & 36 & \\
Female & 19 & 6 & 13 & $<0.01$ \\
TNM stage & & & & \\
Ta-T1 & 41 & 20 & 21 & $<0.01$ \\
T2-T4 & 33 & 4 & 29 & \\
Grade & & & & \\
LG & 23 & 15 & 8 & \\
HG & 51 & 10 & 41 & \\
\hline
\end{tabular}

CMTM8 expression in bladder cancer tissues from patients was categorized based on tumor stage and grade, and analyzed by immunohistochemistry. Scoring criteria protocol was followed as mentioned in Materials and methods.

CMTM8 plasmid in the EJ cells compared to the empty-vector and wild-type cells (Fig. 2D). Furthermore, confirmation of the overexpression of CMTM8 was achieved when GFP was detected as green fluorescence under a fluorescence microscope, indicating efficient transfection of the overexpressing plasmid in both the T24 and EJ cell lines (Fig. 2E and F). These results provide evidence that we were able to successfully overexpress CMTM8 in the bladder cancer cell lines, which were utilized in subsequent experiments to understand the impact of this tumor-suppressor protein in different bladder cancer cell lines.

Overexpression of CMTM8 impacts the cell viability and the colony numbers in bladder cancer cells. As revealed by the CCK-8 assay, overexpression of CMTM8 in T24 cells resulted in overall decreased cell viability compared to the cell viability of the wild-type and empty-vector control over a period of 6 days (Fig. 3A). Cell viability was slower, with the lowest level on day 4, after which, it regained growth slightly until the end of day 6 . In the case of EJ bladder cancer cells, the decline in cell growth was most noticeable in cells transfected with the CMTM8 vector compared to the wild-type and empty vector-transfected cells (Fig. 3B). The trend was almost linear with a uniform elevation in growth until the endpoint on day 5. In both cell lines, the wild-type or empty vector-transfected cells showed uniform growth over the period of 5-6 days. This clearly indicates the effect of CMTM8 expression on cell growth. Similar effects were also noted in the colony numbers in cells overexpressing CMTM8. As shown in Fig. 3C, the number of colonies were the same for the wild-type and empty vector-transfected T2 4 cells, while CMTM8 overexpression led 
A

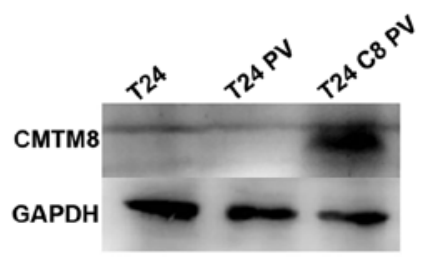

B

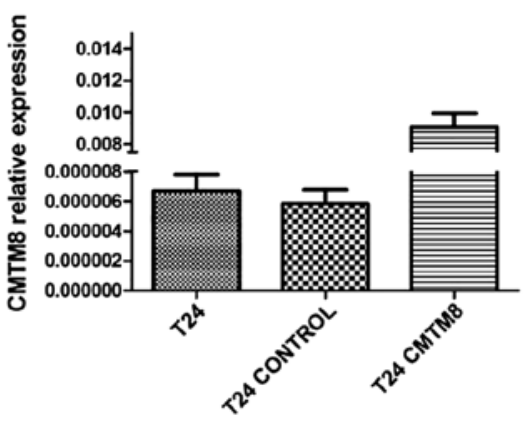

C

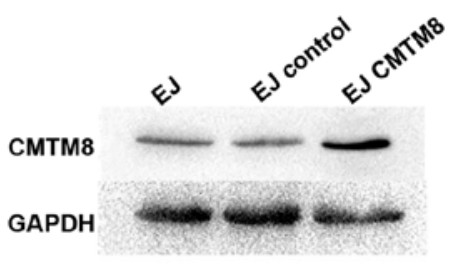

D

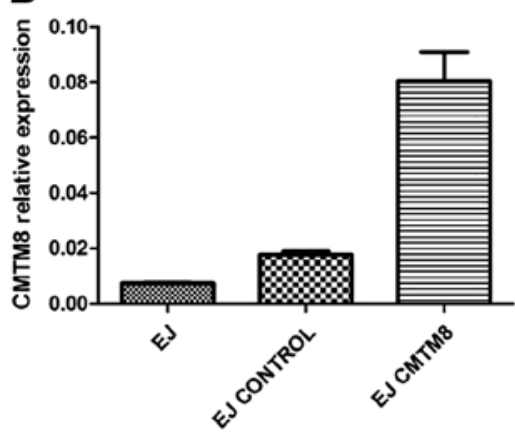

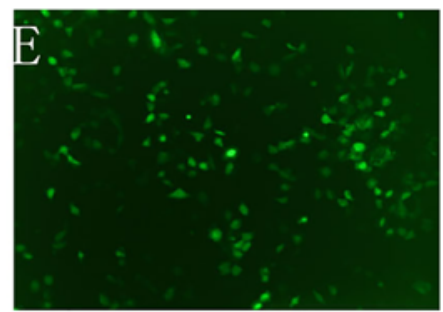

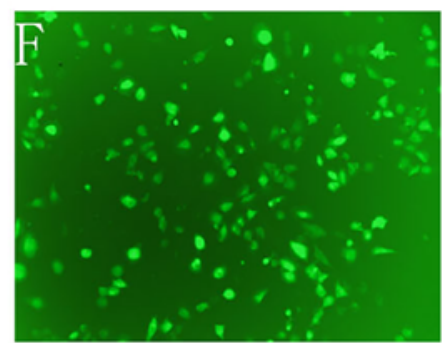

Figure 2. Ectopic CMTM8 overexpression in T24/EJ cell lines. Experiments to induce overexpression were performed by lentiviral transfection. Forty-eight hours after transfection, the cell lines were confirmed to have overexpression of CMTM8 by several techniques. (A) Western blotting showed normal as well as overexpression of CMTM8 in the wild-type (T24) cells and empty vector (T24 PV) or CMTM8-GFP-transfected (T24 C8 PV) cells. (B) mRNA expression of CMTM8 was confirmed by qPCR in the above transfected cells. (C) EJ cells were used for western blotting experiments, which demonstrated low or high expression of CMTM8 in the different cells. (D) mRNA expression of CMTM8 in EJ cells transfected with the various plasmids as described earlier. Representative images from GFP-tagged CMTM8-transfected cells showing increased green fluorescence in the (E) T24 and (F) EJ cell lines observed under a fluorescence microscope.

A

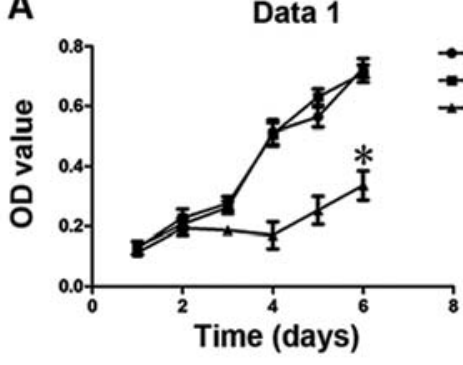

B

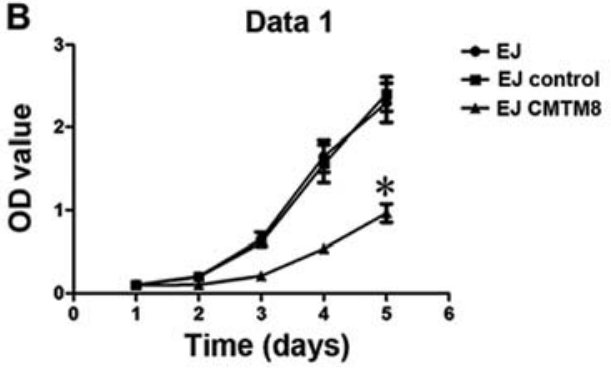

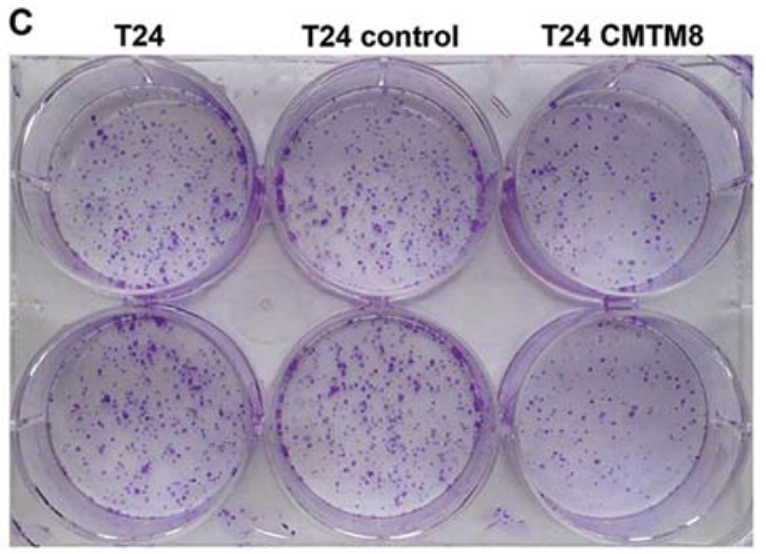

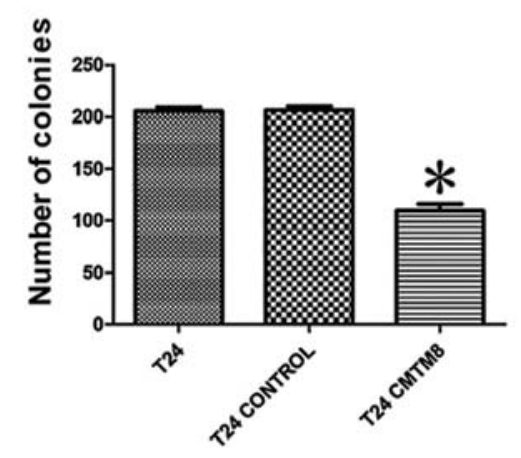

Figure 3. Overexpression of CMTM8 decreases cell proliferation and colony forming ability. (A) CCK-8 assays were performed for a period of $144 \mathrm{~h}$, in wild-type T24 cells or cells transfected with either the empty vector or the CMTM8-overexpressing plasmid, and the effects on cell viability were assessed. (B) CCK-8 assays were performed in EJ cells with specific transfection plasmids as indicated. (C) Representative images of the colony formation potential of wild-type T24 cells or cells transfected with the empty vector or CMTM8-overexpressing plasmid. Quantitative representation of the colony counts is shown in the right panel. The experiments indicated that CMTM8 overexpression decreased cell and colony growth of both T24 and EJ cells.

to decreased colony growth after 14 days. Likewise, decreased colony growth was also observed in the EJ cells overexpressing
CMTM8 (data not shown). The quantitative estimation of T24 colony numbers revealed that colony number decreased to as 

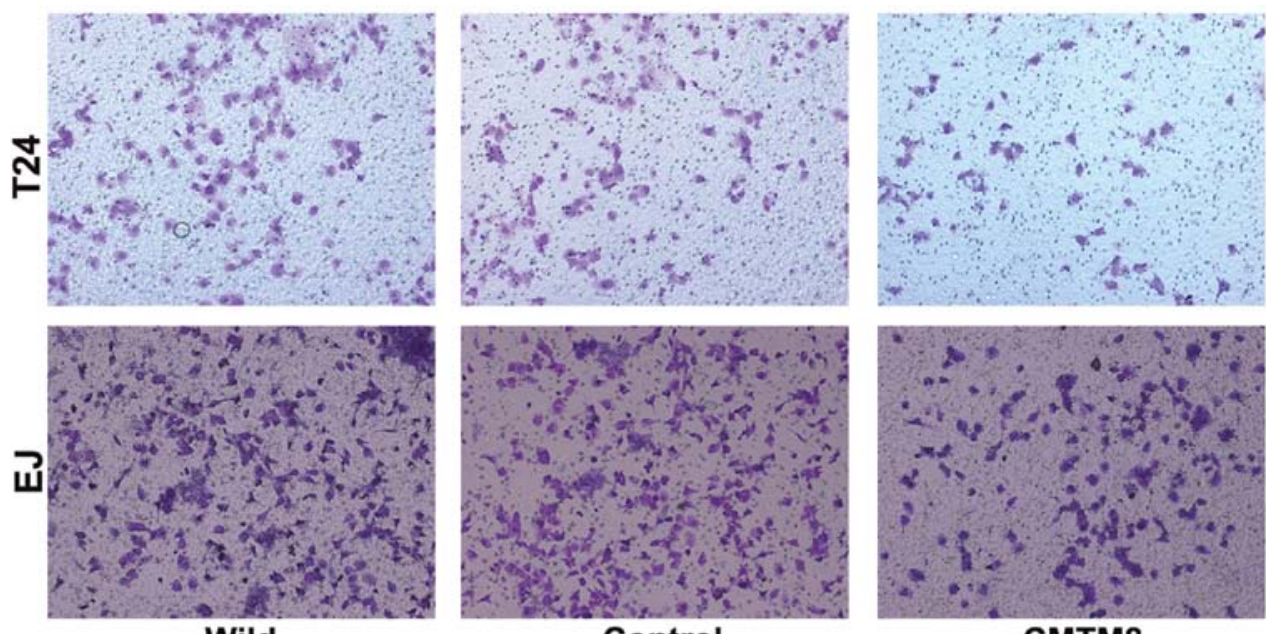

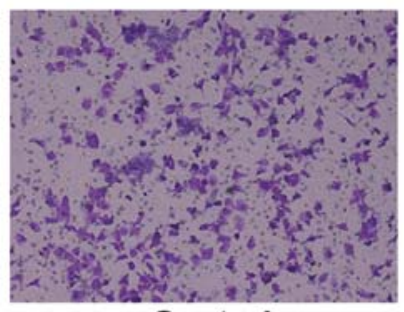

Control

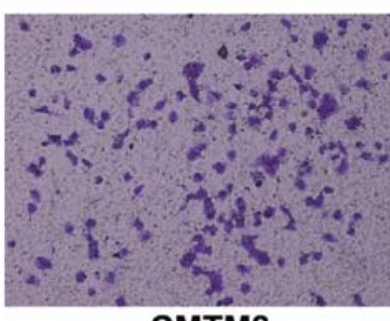

CMTM8
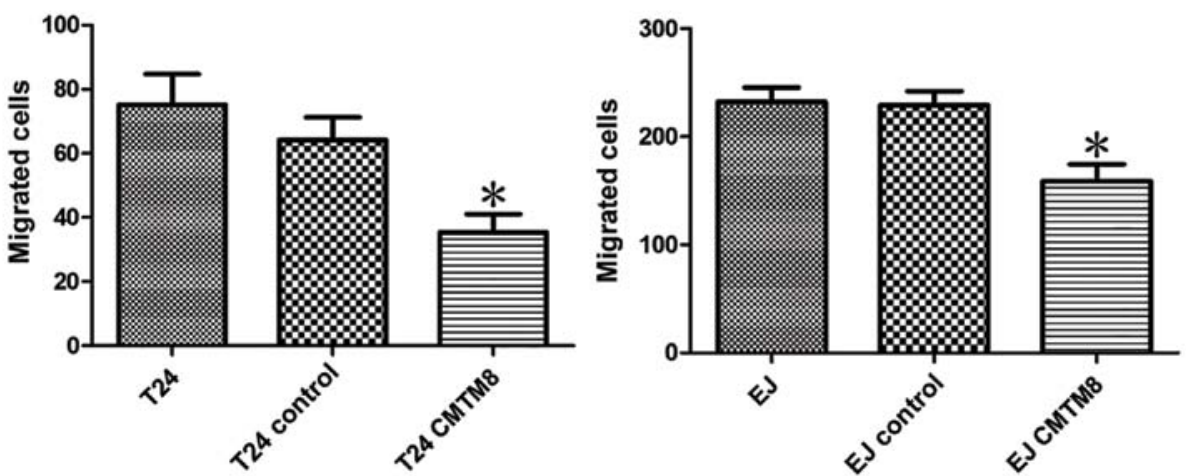

Figure 4. Upregulation of CMTM8 inhibits the migration of bladder cancer cells. Representative images of T24 and EJ cells transfected with the indicated plasmids as described in Materials and methods. The migratory ability of T24 and EJ cells following the different treatments was analyzed by Transwell assays. Quantitative analysis (bottom panel) of the migratory cells indicated that CMTM8-overexpressing T24 cells had 50\% reduced migration less than that of the control or empty vector-transfected cells, while it was $25 \%$ less in the EJ cells. Data represent the mean \pm SEM from three independent experiments. Statistical significant differences were noted between T24/EJ CMTM8-overexpressing cells and wild-type/empty vector-transfected cells ( $\mathrm{P}<0.01$ vs. the other groups).

little as 100 per plate, while it was more than double for the empty-vector/wild-type cells (Fig. 3C, right panel). Hence, it is obvious that overexpression of CMTM8 has marked effects on cell viability and colony formation ability in bladder cancer cells.

Upregulation of CMTM8 inhibits migration and invasion of bladder cancer cells. To determine whether overexpression of CMTM8 has any influence on the migratory ability of T24 and EJ bladder cancer cells, migration Transwell assays were performed. Migratory ability was inhibited in both the T24 and EJ cells transfected with the overexpressing CMTM8 plasmids, while the wild-type and the empty vector-transfected cells showed extensive migration capabilities (Fig. 4). Of note, T24 cells with upregulated CMTM8 showed almost 50\% inhibition in the migratory capacity compared to the respective controls, while the EJ cells overexpressing CMTM8 showed only $30 \%$ migration inhibition compared to the wild-type or empty vector-transfected cells as obtained by the quantitative data $(\mathrm{P}<0.01$ vs. respective controls in both cell lines). This clearly proves that upregulation of CMTM8 is associated with the decreased migratory ability of these cells.

Similarly, the invasion capabilities of these bladder cancer cell lines were assessed using a Matrigel Transwell assay. Representative images revealed that overexpression of CMTM8 inhibited the invasion potential of the T24 and EJ cells (Fig. 5). Quantitative estimation of the invading cells revealed that the inhibition rates achieved were $\sim 40 \%$ in both T24 and EJ cells with overexpression of CMTM8 compared to the wild-type and empty vector-transfected cell lines $(\mathrm{P}<0.01$ vs. respective controls). These results indicate that CMTM8 is a crucial determinant in controlling the cell viability, migration and invasion potential in bladder cancer cells.

CMTM8 inhibits the growth and metastasis of bladder cancer xenografts. Subcutaneous injections of wild-type, lenti-GFP-NTC or lenti-GFP-CMTM8 T24 cells at three different points in the flanks of the mice promoted the development of palpable tumors as early as 3 days. However, the tumor grow th followed different kinetics for the different groups until the mice were sacrificed after 29 days. Specifically, the tumor sizes of the wild-type and lenti-GFP-NTC group were similar, but were in contrast with the lenti-GFP-CMTM8 group at the end of the experiments (Fig. 6A). Wet weights of the subcutaneous tumors were also different, with a $50 \%$ reduction in the tumor weights in the mice injected with the lenti-GFP-CMTM8-transfected cells (Fig. 6B). Furthermore, the tumor volume was also measured every 3 days. The tumor 


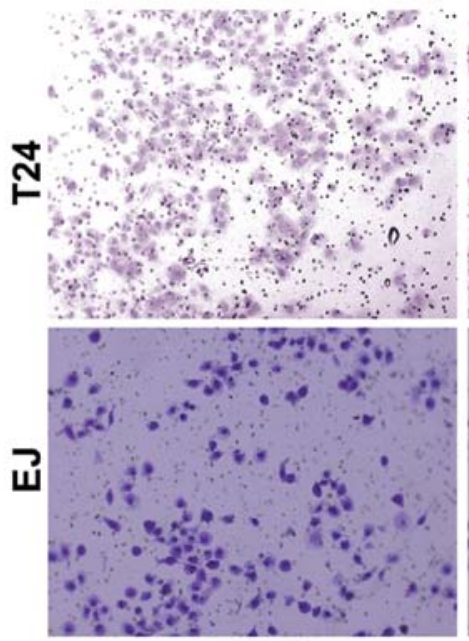

Wild

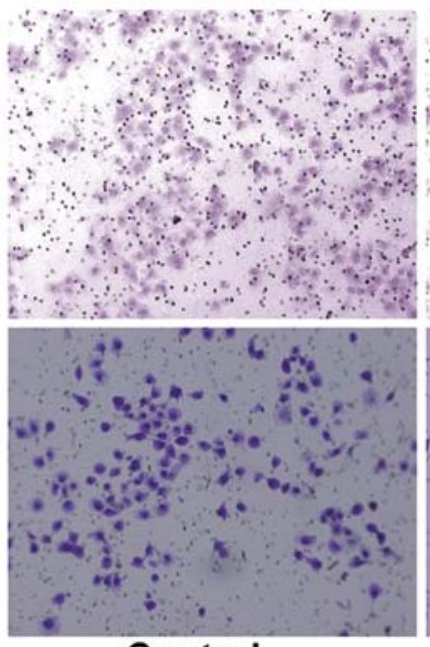

Control

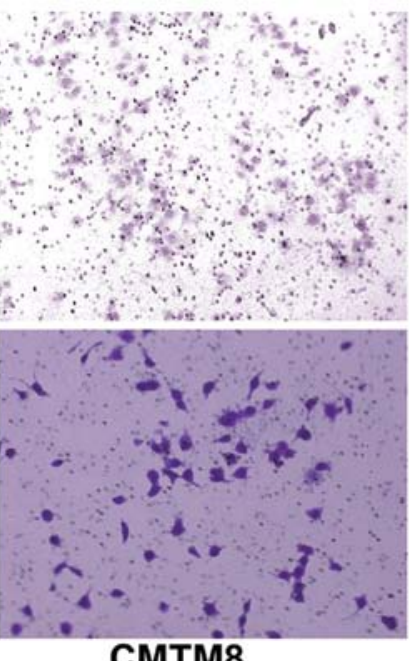

СMTM8
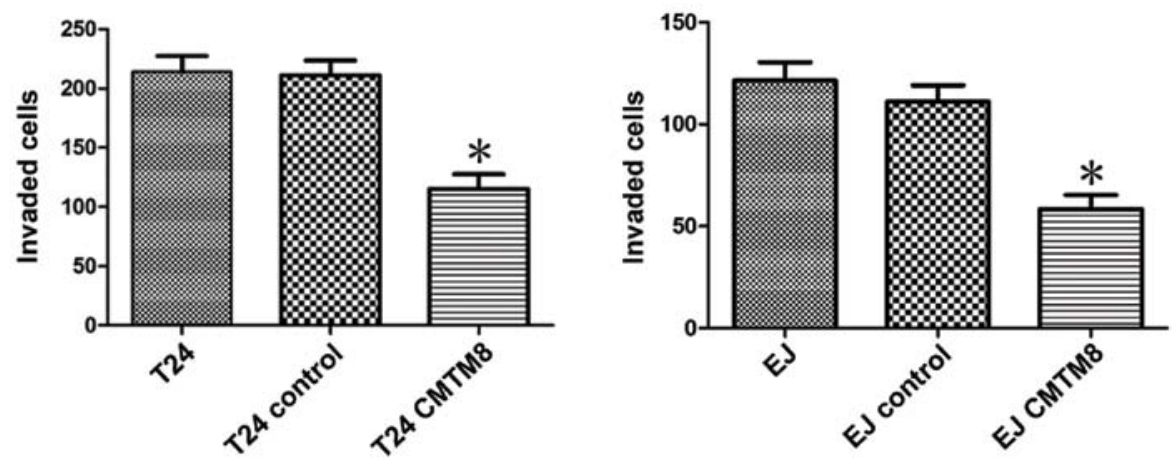

Figure 5. Overexpression of CMTM8 inhibits the invasive potential of bladder cancer cells. Representative images of T24 and EJ cells transfected with the indicated plasmids as described in Materials and methods. Invasive capability of T24 and EJ cells following the different treatments was analyzed on Matrigel-coated membranes. Quantitative analysis (bottom panel) of the invading cells indicated that CMTM8-transfected T24 cells had 50\% reduced invasion on the membranes than the control or empty vector-transfected cells. In the EJ cells overexpressing CMTM8, the invasive ability declined $\sim 60 \%$ as compared to the other groups. Data represent the mean \pm SEM from three independent experiments. Statistical significant differences were obtained between T24/EJ CMTM8-overexpressing cells and wild-type/empty vector-transfected cells ( $\mathrm{P}<0.01$ vs. the other groups).

volumes exhibited increasing trends for the wild-type and NTC group, while tumors had slower growth in the case of CMTM8 mice. It is interesting to note that there were no statistically significant differences in the tumor volumes between groups, until day 15 . However, the tumor volumes measured on day 15 were $\sim 400 \mathrm{~mm}^{3}$ in the control groups, while it showed a reduced volume of $200 \mathrm{~mm}^{3}$ in the CMTM8 mouse group (Fig. 6C). At the end of 27 days, the tumor volumes in the controls increased to $600 \mathrm{~mm}^{3}$, while CMTM8 mice remained at only $300 \mathrm{~mm}^{3}$. Thus, a $50 \%$ reduction in tumor volume was noted in the mice injected with T24 cells expressing CMTM8, compared to the wild-type T24 or NTC-transfected T24 cells. Since the cells were also transfected with GFP, tumor monitoring was performed every two weeks in the NTC and CMTM8 groups by IVIS-Lumina live animal imaging. The intensity of fluorescence from low to high (blue to red) indicates the increasing tumor burden in the animals as pictured on days 15 and 29 (Fig. 7A). The intensities of the tumors as represented by fluorescence were higher in the T24-controls compared to the T24-CMTM8 mice. The intensity of fluorescence as calculated by the ROI, revealed that there was a $30 \%$ reduction in the fluorescence intensity in the CMTM8 mice compared to the NTC mice (Fig. 7B). Next, in order to determine the role of CMTM8 in metastasis, mice were positioned supine, and imaging was performed on day 29. As expected, axillary lymph node metastasis was found in 4 out of 5 mice, while in the CMTM8 mouse group, only 1 out of 5 were affected to a smaller extent (Fig. 7A, bottom panel). The quantitation of lymph node metastasis indicated that the control mice had $\geq 3$ metastases, while this number was only 1 in the case of the CMTM8 group. Thus, these experiments clarify the role of CMTM8 in the prevention of metastasis in bladder cancer.

\section{Discussion}

Bladder cancer is the fourth most common malignancy in males in Western countries with a majority of cases arising from the bladder urothelia $(23,24)$. Primary treatments include transurethral resection of the bladder tumor (TURBT) followed by intravesical chemotherapy, Bacillus Calmette-Guérin immunotherapy or radical cystectomy in advanced cases (24). Disease heterogeneity plays a vital role in the aggressiveness and clinical outcome of this disease, and bladder cancer often displays strong relapse potential (25). Therefore, robust biomarkers and molecular signatures are always in demand that can be utilized to offer 
A
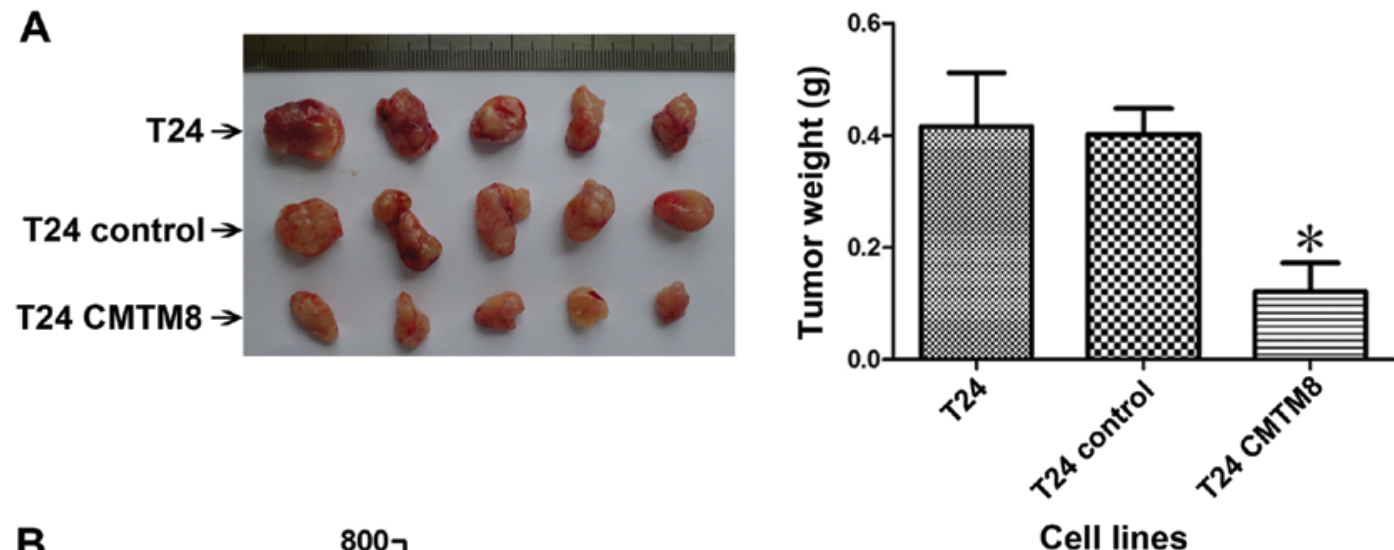

B

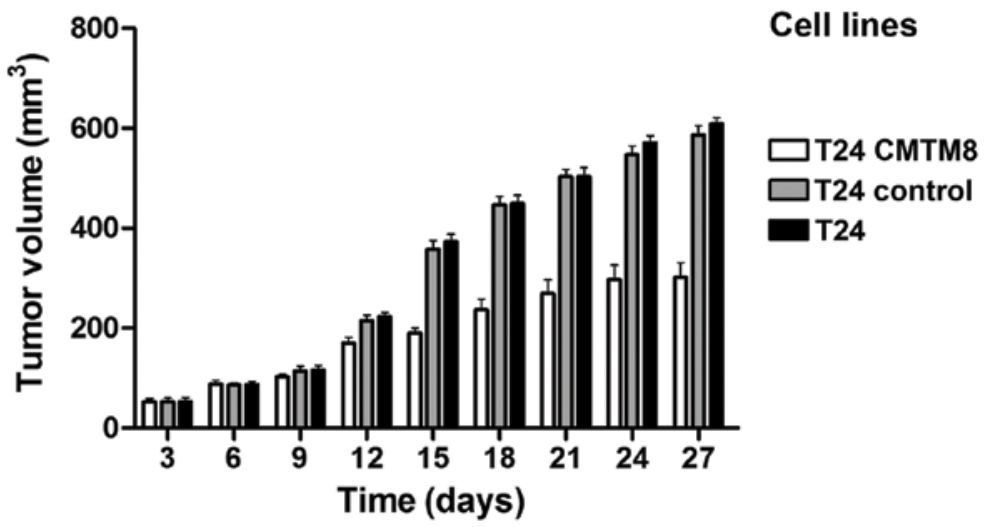

Figure 6. Upregulation of CMTM8 impacts subcutaneous T24 xenograft tumor growth. (A) Comparison of bladder cancer xenograft tumors obtained from cells injected with different plasmids as indicated. Tumor sizes were distinctly different in the control/empty vector vs. CMTM8-overexpressing group. Wet-weights of bladder tissues as soon as they were removed from the skin were measured and plotted as a bar graph (A, right panel). (B) Tumor volumes were measured every 3 days from all the groups as indicated and plotted in the above graph. Data represent the mean \pm SEM from three independent experiments. Statistical significances were obtained at ${ }^{*} \mathrm{P}<0.01$ vs. the other groups.

viable approaches, to improve disease prognosis and treatment. The present study demonstrated the role of a specific tumor-suppressor protein, CMTM8, in bladder cancer. Samples from bladder cancer patients revealed that reduced expression was associated with advanced tumor grade and stage, and vice-versa. We showed that exogenous overexpression of CMTM8 in bladder cancer cells led to a decrease in cell viability, colony forming ability, migratory and invasive potential. Furthermore, decreased tumor growth and metastatic potential was observed in experimental xenograft tumors developed from CMTM8-overexpressing bladder cancer cells.

CMTM is a recently identified MARVEL domaincontaining protein family of proteins with various roles in physiological systems, such as reproduction and immunity. CMTM1, CMTM2 and CMTM3 are predominantly found in testis, while CMTM2a and CMTM2b are needed for androgen receptor function (26-28). CMTM3, CMTM6 and CMTM7 are found in peripheral blood mononuclear cells (PBMCs) indicating their strong association with the immune system (28). CMTM8 plays a pivotal role in tumorigenesis, growth factor receptor endocytosis and apoptosis induction. A previous study demonstrated that overexpression of CMTM8 enhanced EGFR internalization, implicating its role in the inhibition of tumorigenesis (29). EGFR is expressed in most bladder and osteosarcoma tumors, and is often associated with tumor progression and mortality $(30,31)$. Considering the close connection with EGFR, CMTM8 was studied in bladder cancer. The present study investigated the expression profile of CMTM8 in human bladder cancer and its biological roles. A negative correlation between pathological stage/grade and expression of CMTM8 was observed in the patient samples. In support of this finding, low CMTM8 protein and mRNA expression was also evident in bladder cancer cell lines. The expression of CMTM8 has been previously studied in other cancers as well. Overall, CMTM8 has been reported to be downregulated in solid tumors leading to their progression and survival. In HepG2 hepatocellular carcinoma, acquisition of epithelial-to-mesenchymal (ETM) transition was noted in CMTM8-knockdown cells. However, overexpression inhibited EMT-like changes and cell motility (14). Further downregulation of CMTM8 also induced EMT-like morphological changes in non-tumorigenic human breast MCF-10A cells, indicating a prominent role for CMTM8 in cellular transformation (14). This transformation was mainly due to the loss of c-Met inhibition induced by CMTM8, which activated migration, invasion and proliferation of cancer cells $(10,14)$.

In human bladder cancer, $\mathrm{T} 2$ stage tumors invade into the muscle layer, and outgrow into a T3 bladder cancer quite spontaneously when conditions are favorable. High stage bladder cancers are aggressive and invasive, and spread to lymph nodes and other organs, devastating the human body within a few months. Furthermore, bladder carcinoma with a higher grade is more likely to recur and progress faster. In this study, there was a significant negative correlation between expression of CMTM8 and advanced primary tumor stage (T2-T4) 

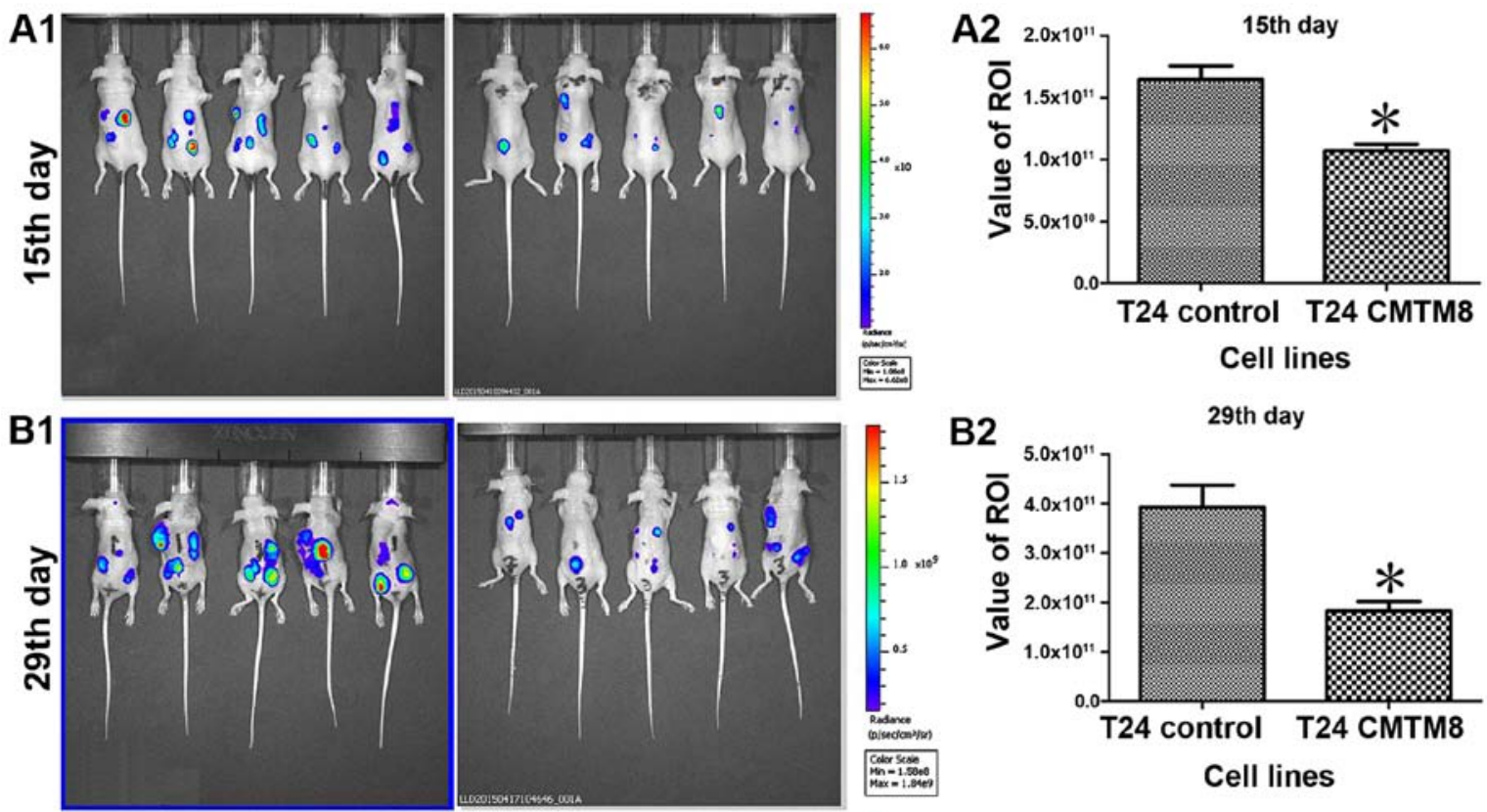

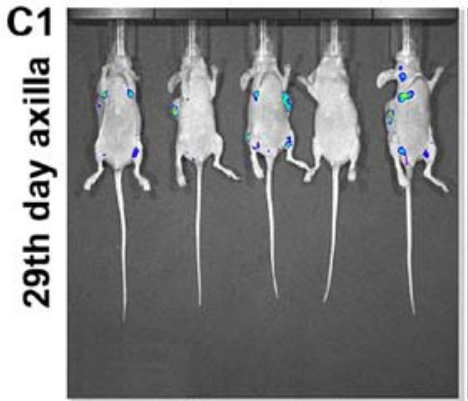

T24 control

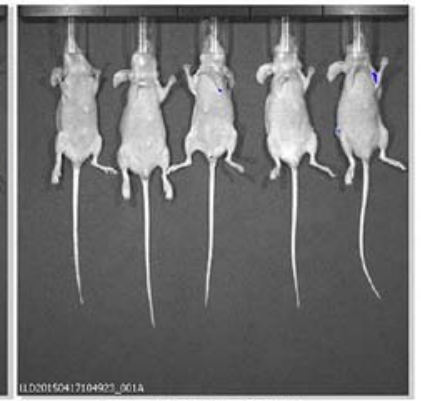

T24 CMTM8
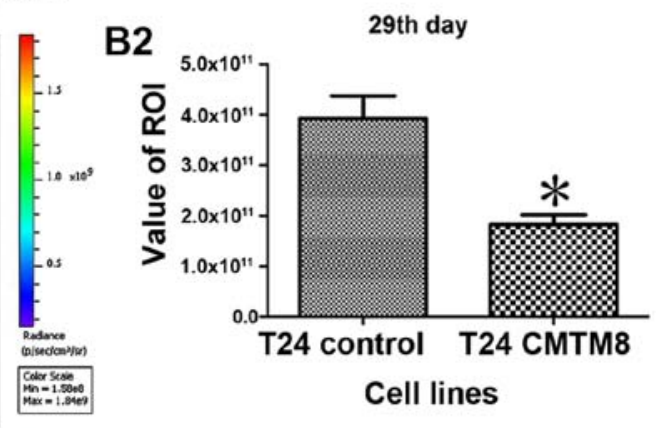

C2
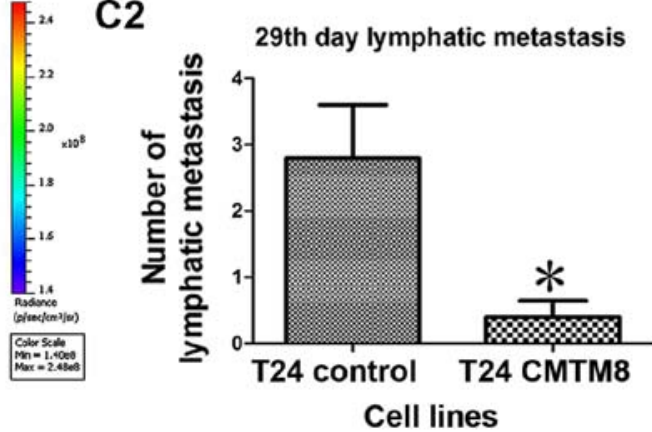

Figure 7. CMTM8-overexpressing T24 xenografts exhibit suppression of tumorigenesis and lymphatic metastasis in vivo. IVIS imaging was utilized to monitor the tumor burden over the entire period of the experiment. (A1) Imaging from day 15 and fluorescence intensity representative of tumor burden (blue to red represents low to high intense tumors) is shown. Animals injected with T24 cells overexpressing CMTM 8 show reduced tumor formation compared to the controls. Quantification of fluorescence intensity is shown (A2, right panel). (B1) Further imaging was conducted on day 29 as indicated, and demonstrated robust tumor formation in the control animals. Quantification data is shown (B2, right panel). (C1) On day 29, and before termination of the experiments, animals were imaged in a supine position, to reveal axillary metastasis in the majority of the controls. In the CMTM8 mice, axillary metastasis was less evident. Quantitative data on the number of lymphatic metastasis are plotted (C2, right panel). Data represent mean \pm SEM ( $=5 /$ group). Statistical significant differences were obtained at ${ }^{*} \mathrm{P}<0.01$ vs. the control group.

and tumor grade. In a different study, yet another biomarker WWOX was significantly associated with high histological grade and stage of bladder cancer (32). Yet, our study explored the biological effects by overexpressing CMTM8 in bladder cancer cells, which led to the reversal of oncogenic events in these cells. Although biomarkers such as CMTM8 and WWOX acted as tumor suppressors in these studies, a large number of patient samples is needed to delineate the precise roles and arrive at a significant conclusion, which is a main limiting factor in these studies.

Experimental studies on bladder cancer cell lines of varying grade were also conducted to elucidate a non-biased role for CMTM8 in this type of cancer. Our results reflected that low-grade RT4 cells had high expression levels of CMTM8 protein and mRNA. There are chances that CMTM8 being a tumor suppressor was predominantly expressed in these papillary tumors, while there could be unknown factors that suppress the expression of CMTM8 in high-grade bladder cancer cell lines. Our biological studies indicated that overexpression of CMTM8 significantly downregulated all aspects of tumor progression including cell viability, colony formation, migratory and invasive potential of the highly aggressive bladder cancer cell lines. Previous research indicated that CMTM3 overexpression suppressed cell proliferation and anchorage-independent cell growth in clear cell renal cell carcinoma, another type of solid tumor. A possible explanation is the involvement of epigenetic silencing in the inhibition of tumorigenesis (33). Although our studies highlight the significance of CMTM8 as a biomarker, which could also be manipulated to improve the therapeutic outcome in bladder cancer, the mechanism is still unknown at this point. Since MARVEL is a common feature of the Physins, Gyrins, Occludin, MAL and CMTM families, a membrane domain responsible for the apposition events such as membrane transport and trafficking (34), we speculate that EGFR and/or VEGFR related signaling could be involved in the CMTM8 function. A positive correlation of VEGF and bladder tumorigenesis has previously been deduced. Increased VEGF is a prominent feature of progressive bladder tumors as demonstrated by Crew et al, which could be extrapolated to the 
present study in relation to CMTM8 expression (35). Hence, the magnitude of interaction between CMTM8 and VEGF may be able to underscore the relapse potential as well as in the prediction of possible intravesical therapy in aggressive cancers such as bladder cancer.

Next, for the effective translational efficacy of the manipulation of CMTM8 overexpression, our studies utilized bladder cancer xenografts implanted from CMTM8-overexpressing cells. The in vivo imaging studies also revealed that the tumor growth was diminished as observed by the ROI data upon upregulation of CMTM8. Furthermore, metastasis was also limited compared to the control or empty vector-transfected cells. Previous study in HepG2 carcinoma also revealed a negative correlation of CMTM8 and tumor progression via the c-MET/ERK pathways (14). c-MET signaling contributes to the invasive growth and cancer metastasis, driving tumor growth of many cancers beyond their primary sites (36). Hence, it is plausible to infer that CMTM8 overexpression blocked c-MET signaling in our in vivo models of bladder cancer, once again reaffirming the potential of CMTM8 as a tumor suppressor.

In summary, we demonstrated that CMTM8 may potentially be developed as a novel biomarker with clinical predictive and prognostic values in bladder cancer. Advanced tumor stage and grade were reciprocally correlated with the expression of CMTM8 in the bladder cancer tissues and cell lines. Overexpression of this protein significantly reversed the events associated with bladder tumorigenesis in vitro as well as in vivo. Although, more robust and randomized studies are warranted, this study revealed the tumor-suppressor role of CMTM8 in bladder cancer.

\section{Acknowledgements}

This study was supported by the National Natural Science Foundation of China (NSFC 81272830). We thank Dr Liqun Zhou at the Peking University Institute of Urology for providing the EJ bladder cancer cell line.

\section{References}

1. Torre LA, Bray F, Siegel RL, Ferlay J, Lortet-Tieulent J and Jemal A: Global cancer statistics, 2012. CA Cancer J Clin 65: 87-108, 2015.

2. Burger M, Catto JW, Dalbagni G, Grossman HB, Herr H, Karakiewicz P, Kassouf W, Kiemeney LA, La Vecchia C, Shariat $\mathrm{S}$, et al: Epidemiology and risk factors of urothelial bladder cancer. Eur Urol 63: 234-241, 2013.

3. Guillaume L and Guy L: Epidemiology of and risk factors for bladder cancer and for urothelial tumors. Rev Prat 64: 1372-1374, 1378-1380, 2014 (In French).

4. Almeida M, Canas-Marques R, Lopez-Beltran A, Rebola J, Lúcio R, Montironi R, Cheng L and Fonseca J: Small cell carcinoma of the bladder associated with schistosomiasis: A case report. Anal Quant Cytopathol Histpathol 36: 339-344, 2014.

5. Kumagai T, Takeyama N, Yabuki T, Harada M, Miki Y, Kanou H, Inoue S, Nakagawa T and Noguchi H: Apheresis of activated leukocytes with an immobilized polymyxin B filter in patients with septic shock. Shock 34: 461-466, 2010.

6. Babjuk M, Burger M, Zigeuner R, Shariat SF, van Rhijn BW, Compérat E, Sylvester RJ, Kaasinen E, Böhle A, Palou Redorta J, et al; European Association of Urology: EAU guidelines on non-muscle-invasive urothelial carcinoma of the bladder: Update 2013. Eur Urol 64: 639-653, 2013.

7. Millán-Rodríguez F, Chéchile-Toniolo G, Salvador-Bayarri J, Palou J, Algaba F and Vicente-Rodríguez J: Primary superficial bladder cancer risk groups according to progression, mortality and recurrence. J Urol 164: 680-684, 2000.
8. Han W, Lou Y, Tang J, Zhang Y, Chen Y, Li Y, Gu W, Huang J, Gui L, Tang Y, et al: Molecular cloning and characterization of chemokine-like factor 1 (CKLF1), a novel human cytokine with unique structure and potential chemotactic activity. Biochem J 357: 127-135, 2001.

9. Han W, Ding P, Xu M, Wang L, Rui M, Shi S, Liu Y, Zheng Y, Chen Y, Yang T, et al: Identification of eight genes encoding chemokine-like factor superfamily members 1-8 (CKLFSF1-8) by in silico cloning and experimental validation. Genomics 81 : 609-617, 2003.

10. Salvi A, Arici B, Portolani N, Giulini SM, De Petro G and Barlati S: In vitro c-met inhibition by antisense RNA and plasmid-based RNAi down-modulates migration and invasion of hepatocellular carcinoma cells. Int J Oncol 31: 451-460, 2007.

11. Polyak K and Weinberg RA: Transitions between epithelial and mesenchymal states: Acquisition of malignant and stem cell traits. Nat Rev Cancer 9: 265-273, 2009.

12. Hu H, Chen JW, Xu KX, Wang D, Wang Y, Wang GW, Zhang SY and Wang XF: Expressions of CMTM8 and E-cadherin in primary and metastatic clear cell renal cell carcinoma. Beijing Da Xue Xue Bao 45: 537-541, 2013 (In Chinese).

13. Choi YK, Woo SM, Cho SG, Moon HE, Yun YJ, Kim JW, Noh DY, Jang BH, Shin YC, Kim JH, et al: Brain-metastatic triple-negative breast cancer cells regain growth ability by altering gene expression patterns. Cancer Genomics Proteomics 10: 265-275, 2013.

14. Zhang W, Mendoza MC, Pei X, Ilter D, Mahoney SJ, Zhang Y, Ma D, Blenis J and Wang Y: Down-regulation of CMTM8 induces epithelial-to-mesenchymal transition-like changes via c-MET/extracellular signal-regulated kinase (ERK) signaling. J Biol Chem 287: 11850-11858, 2012.

15. Chen SQ, Du Y, Huang YM, Wang X, Gu QL and Dong ZM: Effects of novel human chemokine-like factor superfamily 8 on proliferation and EGFR expression of HL-60 cells. Zhongguo Shi Yan Xue Ye Xue Za Zhi 15: 458-461, 2007 (In Chinese).

16. Jin C, Ding P, Wang Y and Ma D: Regulation of EGF receptor signaling by the MARVEL domain-containing protein CKLFSF8. FEBS Lett 579: 6375-6382, 2005.

17. Jin C, Wang Y, Han W, Zhang Y, He Q, Li D, Yin C, Tian L, Liu D, Song Q, et al: CMTM8 induces caspase-dependent and -independent apoptosis through a mitochondria-mediated pathway. J Cell Physiol 211: 112-120, 2007.

18. Black PC and Dinney CP: Growth factors and receptors as prognostic markers in urothelial carcinoma. Curr Urol Rep 9: 55-61, 2008.

19. Mellon K, Wright C, Kelly P, Horne CH and Neal DE: Long-term outcome related to epidermal growth factor receptor status in bladder cancer. J Urol 153: 919-925, 1995.

20. Kassouf W, Black PC, Tuziak T, Bondaruk J, Lee S, Brown GA, Adam L, Wei C, Baggerly K, Bar-Eli M, et al: Distinctive expression pattern of ErbB family receptors signifies an aggressive variant of bladder cancer. J Urol 179: 353-358, 2008.

21. Chow NH, Liu HS, Lee EI, Chang CJ, Chan SH, Cheng HL, Tzai TS and Lin JS: Significance of urinary epidermal growth factor and its receptor expression in human bladder cancer. Anticancer Res 17: 1293-1296, 1997.

22. Fu L, Liu N, Han Y, Xie C, Li Q and Wang E: ADAM10 regulates proliferation, invasion, and chemoresistance of bladder cancer cells. Tumour Biol 35: 9263-9268, 2014.

23. Jemal A, Bray F, Center MM, Ferlay J, Ward E and Forman D: Global cancer statistics. CA Cancer J Clin 61: 69-90, 2011.

24. Prasad SM, Decastro GJ and Steinberg GD; Medscape: Urothelial carcinoma of the bladder: Definition, treatment and future efforts. Nat Rev Urol 8: 631-642, 2011.

25. Avgeris M, Mavridis K, Tokas T, Stravodimos K, Fragoulis EG and Scorilas A: Uncovering the clinical utility of miR-143, miR-145 and miR-224 for predicting the survival of bladder cancer patients following treatment. Carcinogenesis 36: 528-537, 2015.

26. Wang L, Wu C, Zheng Y, Qiu X, Wang L, Fan H, Han W, Lv B, Wang Y, Zhu X, et al: Molecular cloning and characterization of chemokine-like factor super family member 1 (CKLFSF1), a novel human gene with at least 23 alternative splicing isoforms in testis tissue. Int J Biochem Cell Biol 36: 1492-1501, 2004.

27. Shi S, Rui M, Han W, Wang Y, Qiu X, Ding P, Zhang P, Zhu X, Zhang Y, Gan Q, et al: CKLFSF2 is highly expressed in testis and can be secreted into the seminiferous tubules. Int J Biochem Cell Biol 37: 1633-1640, 2005. 
28. Zhong J, Wang Y, Qiu X, Mo X, Liu Y, Li T, Song Q, Ma D and Han W: Characterization and expression profile of CMTM3/CKLFSF3. J Biochem Mol Biol 39: 537-545, 2006.

29. Li D, Jin C, Yin C, Zhang Y, Pang B, Tian L, Han W, Ma D and Wang Y: An alternative splice form of CMTM8 induces apoptosis. Int J Biochem Cell Biol 39: 2107-2119, 2007.

30. Neal DE, Marsh C, Bennett MK, Abel PD, Hall RR, Sainsbury JR and Harris AL: Epidermal-growth-factor receptors in human bladder cancer: Comparison of invasive and superficial tumours. Lancet 1: 366-368, 1985.

31. Both J, Krijgsman O, Bras J, Schaap GR, Baas F, Ylstra B and Hulsebos TJ: Focal chromosomal copy number aberrations identify CMTM8 and GPR177 as new candidate driver genes in osteosarcoma. PLoS One 9: e115835, 2014.

32. Płuciennik E, Nowakowska M, Stępien A, Wołkowicz M, Stawiński A, Różański W, Lipiński M and Bednarek AK: Alternating expression levels of WWOX tumor suppressor and cancer-related genes in patients with bladder cancer. Oncol Lett 8 2291-2297, 2014.
33. Xie J, Yuan Y, Liu Z, Xiao Y, Zhang X, Qin C, Sheng Z, Xu T and Wang X: CMTM3 is frequently reduced in clear cell renal cell carcinoma and exhibits tumor suppressor activities. Clin Transl Oncol 16: 402-409, 2014.

34. Sánchez-Pulido L, Martín-Belmonte F, Valencia A and Alonso MA: MARVEL: A conserved domain involved in membrane apposition events. Trends Biochem Sci 27: 599-601, 2002.

35. Crew JP, O'Brien T, Bradburn M, Fuggle S, Bicknell R, Cranston D and Harris AL: Vascular endothelial growth factor is a predictor of relapse and stage progression in superficial bladder cancer. Cancer Res 57: 5281-5285, 1997.

36. Trusolino L, Bertotti A and Comoglio PM: MET signalling: Principles and functions in development, organ regeneration and cancer. Nat Rev Mol Cell Biol 11: 834-848, 2010. 